\title{
Shape Preserving Data Interpolation Using Rational Cubic Ball Functions
}

\author{
Ayser Nasir Hassan Tahat, ${ }^{1}$ Abd Rahni Mt Piah, ${ }^{1}$ and Zainor Ridzuan Yahya ${ }^{2}$ \\ ${ }^{1}$ School of Mathematical Sciences, Universiti Sains Malaysia (USM), 11800 Pulau, Malaysia \\ ${ }^{2}$ Institut Matematik Kejuruteraan, Universiti Malaysia Perlis, Kampus Pauh Putra, 02600 Arau, Perlis, Malaysia
}

Correspondence should be addressed to Ayser Nasir Hassan Tahat; aysertahat@yahoo.com

Received 8 April 2015; Accepted 9 June 2015

Academic Editor: Juan Manuel Peña

Copyright (C) 2015 Ayser Nasir Hassan Tahat et al. This is an open access article distributed under the Creative Commons Attribution License, which permits unrestricted use, distribution, and reproduction in any medium, provided the original work is properly cited.

\begin{abstract}
A smooth curve interpolation scheme for positive, monotone, and convex data is developed. This scheme uses rational cubic Ball representation with four shape parameters in its description. Conditions of two shape parameters are derived in such a way that they preserve the shape of the data, whereas the other two parameters remain free to enable the user to modify the shape of the curve. The degree of smoothness is $C^{1}$. The outputs from a number of numerical experiments are presented.
\end{abstract}

\section{Introduction}

Interpolation is a fundamental process in scientific visualization. Smooth curve representation is significant in visualizing the scientific data of various areas of scientific research, including scientific visualization, computer graphics, geometric modeling, numerical analysis, approximation theory, and other fields [1]. In particular, when the data are obtained from complex functions or scientific phenomena, incorporating the inherent features of the data becomes crucial. Smoothness is also a crucial requirement for a pleasing visual display [2]. In recent years, interpolation problems by using splines have attracted growing interest. In many interpolation problems, the solution that preserves some shape properties, such as positivity, monotonicity, and convexity, is important. Many physical situations have entities that gain meaning only when their values appear in positive, monotonic, or convex shape. Therefore, discussing shape preserving interpolation problems is important to provide a computationally economical and visually pleasing solution to the problems of different scientific phenomena [3].

Positivity is one of the features of shape. Many physical situations have entities that gain meaning only when their values are positive, such as a probability distribution function, samples of populations, dissemination rate of drugs in the blood, and half-life of a radioactive substance. The problem of positivity preserving interpolation has been addressed by many researchers. Hussain et al. [3] discussed a local shape preserving interpolation scheme for $2 \mathrm{D}$ data by using a piecewise rational quadratic function. Sarfraz and Hussain [1] have used a rational cubic interpolant with two families of shape parameters to obtain $C^{1}$ positivity, monotonicity, and convexity preserving interpolatory spline curves. However, no free shape parameters are provided for the user to adjust the curves further if needed. $A C^{1}$ piecewise rational cubic function has been developed by Sarfraz et al. [4] to preserve the shape of positive data. The interpolant involves four shape parameters in its construction. Two of the shape parameters were constrained to preserve the shape of the data, whereas the other two shape parameters have to be utilized to modify the shape of the curve to the user's liking. Tahat et al. [5] constructed a $C^{1}$ rational cubic Ball interpolant with four shape parameters to preserve the shape of positive and constrained data. Two of the shape parameters are constrained to preserve the shape of the data, whereas the remaining two parameters provide the user with a degree of freedom to adjust the shape of the generated curve. 
Monotone data arise in many physical phenomena, engineering problems, and scientific applications [6]. The result of the tensile strength of a material in engineering is an example of monotone data. The tensile strength of a material can be defined as the maximum force that a material can withstand before breaking. The force applied is usually called stress and is studied alongside the stretch of the material, referred to as strain. The data from these two entities are always monotone. Other examples are the erythrocyte sedimentation rate in cancer patients and the blood uric acid in patients suffering from gout. The digital-to-analog converter is a good example of a monotone data generator. If the device is considered to be monotone, the analog output must also increase as the input code increases in value [6].

The problem of monotonicity (i.e., when the data set is monotone, the interpolant should also preserve monotonicity) has been discussed by various authors. Delbourgo and Gregory [8] developed a piecewise rational cubic interpolation to preserve the monotonicity of monotone data. Hussain and Sarfraz [7] used rational cubic function with four free parameters to preserve the shape of monotone data; two parameters are constrained, whereas the other two remain free for the user to refine the shape of the curve. Tian [9] preserved the shape of monotone data by a $C^{1}$ piecewise rational cubic spline. Piah and Unsworth [10] improved the sufficient conditions derived in [11]. To preserve monotonicity, a rational cubic Ball interpolant was developed by Piah and Unsworth [12] with two shape parameters that can be used to generate the desired monotone curves. However, no flexibility is provided for the user to refine the curves further if needed, so it is unsuitable for interactive curve design.

Convexity is an important shape property and plays a major role in different disciplines and applications. Nonlinear programming in engineering and scientific applications such as design, optimal control, parameter estimation, and approximation of functions are examples of them [13]. The problem of preserving convexity has been examined by many researchers. Brodlie and Butt [14] developed a $C^{1}$ convexity preserving scheme for $2 \mathrm{D}$ data. They divided each interval where the shape of the data was lost into two subintervals by inserting an extra knot so that the shape of the data was preserved. The piecewise cubic interpolant was used to interpolate the data over each subinterval. Sarfraz [15] used a rational cubic Hermite interpolant with one shape parameter to obtain a $C^{l}$ convexity preserving curve method. He derived data dependent constraints on the shape parameter to preserve the shape of the data. He also introduced a rational cubic interpolant with two families of shape parameters to obtain a $C^{1}$ positivity or convexity preserving interpolatory curve, or both [16]. A local shape preserving interpolation method for $2 \mathrm{D}$ data was discussed by Hussain et al. using a piecewise rational (quadratic/linear) function [3]. Sarfraz and Hussain [1] utilized a rational cubic interpolant with two shape parameters, where the shape constraints are derived on the shape parameters to guarantee the shape preservation of the data. Sarfraz et al. [4] presented a $C^{1}$ piecewise rational cubic function to preserve the shape of positive, monotone, and convex data. The interpolant has four free parameters in its description. Hussain et al. [17] preserved the shape of the data by a $C^{1}$ piecewise rational cubic function. Data dependent conditions were derived on the shape parameters to preserve the shape of the data.

In the present study, a $C^{1}$ rational cubic Ball interpolant with four shape parameters is used to solve the problem of positivity, monotonicity, and convexity. The rest of this paper is organized as follows. First, the rational cubic Ball function is described. Second, a derivative approximation scheme is determined. Then, we discuss the problem of developing an interpolating scheme that preserves the shape of positive, monotone, and convex data, followed by the outputs from a number of numerical experiments. Finally, conclusions are presented.

\section{Rational Cubic Ball Function}

Rational spline interpolation is superior to polynomial spline interpolation because the former can carry more degrees of freedom in its description. This freedom can be used to achieve real-life purposes and objectives in various disciplines. In this section, the rational cubic function is introduced with its four shape parameters in its description. These shape parameters can be used to preserve the shape of the data. Cubic Ball basis functions were first introduced by Ball [18] in his lofting surface program CONSURF at the British Aircraft Corporation. These basis functions are as follows:

$$
\begin{aligned}
& s_{0}(x)=(1-t)^{2} \\
& s_{1}(x)=2 t(1-t)^{2} ; \\
& s_{2}(x)=2 t^{2}(1-t) ; \\
& s_{3}(x)=t^{2}
\end{aligned}
$$

Subsequently, two types of generalized Ball basis functions and their corresponding curves were derived in higher degree by Guojin [19] and Said [20], respectively. The shape preserving properties of the generalized Ball basis were discussed by Goodman and Said $[21,22]$. The rational cubic Ball function is described as follows.

Let $\left\{\left(x_{i}, f_{i}\right), i=1,2, \ldots, n\right\}$ be a given set of data points, where $x_{1}<x_{2}<\cdots<x_{n}$. Also, let $h_{i}=x_{i+1}-x_{i}$ and $\theta=$ $\left(x-x_{i}\right) / h_{i}, 0<\theta<1$.

Consider the following interpolating curve $s(x)$ on the interval $\left[x_{i}, x_{i+1}\right]$ :

$$
\begin{aligned}
& s(x) \\
& \equiv s_{i}(x)=\frac{P_{i}(\theta)}{Q_{i}(\theta)} \\
& =\frac{\alpha_{i} f_{i}(1-\theta)^{2}+V_{i}(1-\theta)^{2} \theta+W_{i}(1-\theta) \theta^{2}+\beta_{i} f_{i+1} \theta^{2}}{\alpha_{i}(1-\theta)^{2}+a_{i}(1-\theta)^{2} \theta+b_{i}(1-\theta) \theta^{2}+\beta_{i} \theta^{2}}, \\
& i=1, \ldots, n-1,
\end{aligned}
$$

with

$$
\begin{aligned}
& V_{i}=a_{i} f_{i}+\alpha_{i} h_{i} d_{i} \\
& W_{i}=b_{i} f_{i+1}-\beta_{i} h_{i} d_{i+1} .
\end{aligned}
$$


The rational function $s(x)$ has the following interpolation properties:

$$
\begin{gathered}
s\left(x_{i}\right)=f_{i}, \\
s\left(x_{i+1}\right)=f_{i+1}, \\
s^{\prime}\left(x_{i}\right)=d_{i}, \\
s^{\prime}\left(x_{i+1}\right)=d_{i+1},
\end{gathered}
$$

where $s^{\prime}(x)$ denotes the first derivative of $s$ with respect to $x$ and $d_{i}$ denotes the derivative value (given or estimated) at knot $x_{i}$. Thus, the function $s(x) \in C^{1}\left[x_{0}, x_{n}\right]$ has $\alpha_{i}, \beta_{i}, a_{i}$, and $b_{i}$ as free parameters in the interval $\left[x_{i}, x_{i+1}\right]$.

\section{Derivative Determination}

In most applications, the derivative parameters $d_{i}$ are not given, and therefore we have to identify them either from the given data $\left(x_{i}, f_{i}\right), i=1,2, \ldots, n$, or by other means. In this study, the derivative parameters are computed from the given data in such a way that the $C^{1}$ smoothness of the interpolant (2) is maintained by using the arithmetic mean approximation. Details of this method can be found in [7].

3.1. Arithmetic Mean Method. This is the three-point difference approximation with

$$
d_{i}= \begin{cases}0, & \text { if } \Delta_{i-1}=0 \text { or } \Delta_{i}=0, \\ \frac{\left(h_{i} \Delta_{i-1}+h_{i-1} \Delta_{i}\right)}{h_{i}+h_{i-1}}, & i=2,3, \ldots, n-1,\end{cases}
$$

and the end conditions are given as

$$
\begin{aligned}
& d_{1}= \begin{cases}0 & \text { if } \Delta_{1}=0 \text { or } \operatorname{sgn}\left(d_{1}^{*}\right) \neq \operatorname{sgn}\left(\Delta_{1}\right) \\
d_{1}^{*}=\Delta_{1}+\left(\Delta_{1}-\Delta_{2}\right) \frac{h_{1}}{\left(h_{1}+h_{2}\right)}, & \text { otherwise, }\end{cases} \\
& d_{n}= \begin{cases}0 & \text { if } \Delta_{n-1}=0 \text { or } \operatorname{sgn}\left(d_{n}^{*}\right) \neq \operatorname{sgn}\left(\Delta_{n-1}\right) \\
d_{n}^{*}=\Delta_{n-1}+\left(\Delta_{n-1}-\Delta_{n-2}\right) \frac{h_{n-1}}{\left(h_{n-1}+h_{n-2}\right)}, & \text { otherwise. }\end{cases}
\end{aligned}
$$

\section{Shape Preserving Data Interpolation}

In general, the rational cubic Ball function in (2) does not necessarily preserve the shape inherent in the data. Thus, to attain the shape preserving interpolation, the user may control, on a trial-and-error basis, the shape by modifying the shape parameters in the area where it is not preserved. This method is ineffective and wastes time; thus, sufficient conditions are required for the shape parameters to preserve the inherent features of the data. In this section, we deal with three shape preserving properties, namely, positive, monotone, and convex data interpolation.

4.1. Positive Data Interpolation. For a given set of positive data points with $x_{1}<x_{2}<\cdots<x_{n}$ and $f_{1}>0, f_{2}>0, \ldots$, $f_{n}>0$, the piecewise rational cubic function (2) preserves positivity if $s_{i}(x)>0$. To preserve positivity of $s(x)$, we need to assign suitable values to $\alpha_{i}, \beta_{i}, a_{i}$, and $b_{i}$ in each interval $\left[x_{i}, x_{i+1}\right]$. Strictly positive denominator $Q_{i}(\theta)$ is guaranteed when $\alpha_{i}>0, \beta_{i}>0, a_{i}>0$, and $b_{i}>0$. Thus, the positivity of the interpolant (2) depends on the positivity of cubic polynomial $P_{i}(\theta)$. Thus, the problem reduces to the determination of appropriate values of $a_{i}$ and $b_{i}$ for which the polynomial $P_{i}(\theta)$ is positive. It is clearly seen that $P_{i}(\theta)$ is positive when $V_{i}$ and $W_{i}$ are both positive. From (3) we can say that

$$
\begin{aligned}
V_{i}>0 \quad \text { when } a_{i} & >\frac{-\alpha_{i} h_{i} d_{i}}{f_{i}}, \\
W_{i}>0 & \text { when } b_{i}>\frac{\beta_{i} h_{i} d_{i+1}}{f_{i+1}} .
\end{aligned}
$$

The above discussion can be written in the form of the following theorem.

Theorem 1. The rational cubic polynomial (2) preserves the shape of positive data if the shape parameters satisfy the following conditions:

$$
\begin{array}{ll}
\alpha_{i}>0, & \\
\beta_{i}>0, & \\
a_{i}=e_{i}+\max \left\{0, \frac{-\alpha_{i} h_{i} d_{i}}{f_{i}}\right\}, & e_{i}>0, \\
b_{i}=\sigma_{i}+\max \left\{0, \frac{\beta_{i} h_{i} d_{i+1}}{f_{i+1}}\right\}, & \sigma_{i}>0 .
\end{array}
$$


4.2. Monotone Data Interpolation. Let $\left\{\left(x_{i}, f_{i}\right), i=1,2, \ldots, n\right\}$ be monotone data defined over the interval $[a, b]$ such that

$$
\begin{aligned}
& f_{i}<f_{i+1}, \\
& \Delta_{i}=\frac{f_{i+1}-f_{i}}{h_{i}}>0, \\
& d_{i}>0
\end{aligned}
$$

$$
i=1,2, \ldots, n \text {. }
$$

The rational cubic function (2) preserves monotonicity of the data set if the following condition is satisfied:

$$
s_{i}^{\prime}(x)>0, \quad \forall i=1, \ldots, n,
$$

where

$$
s_{i}^{\prime}(x)=\frac{\sum_{i=0}^{5} V_{i}(1-\theta)^{5-i} \theta}{\left[Q_{i}(\theta)\right]^{2}}
$$

with

$$
\begin{aligned}
V_{0}= & \alpha_{i}^{2} d_{i}, \\
V_{1}= & A_{0}+2 \alpha_{i} b_{i} \Delta_{i}-2 \alpha_{i} \beta_{i}\left(d_{i+1}-\Delta_{i}\right), \\
V_{2}= & \frac{3\left(A_{1}-A_{0}\right)}{2}+\frac{A_{4}-A_{5}}{2}+b_{i}\left(a_{i} \Delta_{i}-\alpha_{i} d_{i}\right) \\
& -\beta_{i} a_{i} d_{i+1}+2 \alpha_{i} \beta_{i} \Delta_{i}, \\
V_{3}= & \frac{A_{1}-A_{0}}{2}+\frac{3\left(A_{4}-A_{5}\right)}{2}+b_{i}\left(a_{i} \Delta_{i}-\alpha_{i} d_{i}\right) \\
& -\beta_{i} a_{i} d_{i+1}+2 \alpha_{i} \beta_{i} \Delta_{i}, \\
V_{4}= & A_{5}+2 a_{i} \beta_{i} \Delta_{i}-2 \beta_{i} \alpha_{i}\left(d_{i}-\Delta_{i}\right), \\
V_{5}= & \beta_{i}^{2} d_{i+1} .
\end{aligned}
$$

The sufficient conditions for monotonicity on $\left[x_{i}, x_{i+1}\right]$ are $V_{i}>0$ for $i=0,1, \ldots, 5$ because the denominator of $s_{i}^{\prime}(x)$ is squared and positive. Now, $V_{i}>0$ for $i=0,1, \ldots, 5$ if the following conditions are satisfied:

$$
\begin{aligned}
& a_{i}>\frac{\alpha_{i}\left(d_{i}-\Delta_{i}\right)}{\Delta_{i}}, \\
& b_{i}>\max \left\{\frac{\beta_{i}\left(d_{i+1}-\Delta_{i}\right)}{\Delta_{i}}, \frac{\beta_{i} a_{i} d_{i+1}}{\left(a_{i} \Delta_{i}-\alpha_{i} d_{i}\right)}\right\} .
\end{aligned}
$$

The preceding discussion can be summarized in the following theorem.

Theorem 2. Let $\left\{\left(x_{i}, f_{i}\right), i=1,2, \ldots, n\right\}$ be a monotone data. The rational cubic polynomial given in (2) preserves the monotonicity of monotone data if the shape parameters satisfy the following conditions:

$$
\begin{aligned}
& \alpha_{i}>0, \\
& \beta_{i}>0, \\
& a_{i}=l_{i}+\frac{\alpha_{i}\left(d_{i}-\Delta_{i}\right)}{\Delta_{i}}, \quad l_{i}>0, \\
& b_{i}=m_{i}+\max \left\{\frac{\beta_{i}\left(d_{i+1}-\Delta_{i}\right)}{\Delta_{i}}, \frac{\beta_{i} a_{i} d_{i+1}}{\left(a_{i} \Delta_{i}-\alpha_{i} d_{i}\right)}\right\}, \\
& m_{i}>0 .
\end{aligned}
$$

4.3. Convex Data Interpolation. Let $\left\{\left(x_{i}, f_{i}\right), i=1,2, \ldots, n\right\}$ be a convex set of data defined over the interval $[a, b]$ such that

$$
\Delta_{1}<\Delta_{2}<\cdots<\Delta_{n-1}
$$

The necessary condition for a convex interpolant $s(x)$ is

$$
d_{1}<\Delta_{1}<\cdots<\Delta_{i-1}<d_{i}<\Delta_{i}<\cdots<\Delta_{n-1}<d_{n}
$$

Now, the rational cubic function (2) preserves the convexity if the following condition is satisfied:

$$
s_{i}^{\prime \prime}(x) \geq 0, \quad \forall i=1, \ldots, n,
$$

where

$$
s_{i}^{\prime \prime}(x)=\frac{\sum_{i=0}^{7} V_{i}(1-\theta)^{7-i} \theta}{h_{i}\left[Q_{i}(\theta)\right]^{3}} .
$$

The denominator of $s_{i}^{\prime \prime}(x)$ is positive if the shape parameters $\alpha_{i}, a_{i}, b_{i}$, and $\beta_{i}$ are positive. The sufficient conditions for convexity on $\left[x_{i}, x_{i+1}\right]$ are $V_{i}>0$ for $i=0,1, \ldots, 7$ and after some simplification, we obtain the following:

$$
\begin{aligned}
V_{0} & =2 \alpha_{i}^{2}\left(b_{i} \Delta_{i}-\left(\beta_{i} d_{i+1}+a_{i} d_{i}\right)+\beta_{i} \Delta_{i}\right), \\
V_{1} & =V_{0}+6 \alpha_{i}^{2}\left(b_{i}\left(\Delta_{i}-d_{i}\right)+\beta_{i}\left(\Delta_{i}-d_{i}\right)\right. \\
& \left.-\beta_{i}\left(d_{i+1}-\Delta_{i}\right)\right), \\
V_{2} & =2\left(V_{1}-V_{0}\right)+6 \alpha_{i}\left(-b_{i}^{2} \Delta_{i}+b_{i} \beta_{i}\left(d_{i+1}-\Delta_{i}\right)\right. \\
& +a_{i} b_{i} \Delta_{i}-b_{i} \beta_{i} \Delta_{i}+\beta_{i}^{2}\left(d_{i+1}-\Delta_{i}\right) \\
& \left.-a_{i} \beta_{i}\left(d_{i+1}-\Delta_{i}\right)+a_{i} \beta_{i} \Delta_{i}+2 \alpha_{i} \beta_{i}\left(\Delta_{i}-d_{i}\right)\right),
\end{aligned}
$$




$$
\begin{aligned}
& V_{3}=\left(V_{2}+V_{0}-V_{1}\right)+2 a_{i}^{2} \beta_{i} \Delta_{i}-2 a_{i} \alpha_{i} \beta_{i}\left(d_{i+1}-\Delta_{i}\right) \\
& +2 \beta_{i}{ }^{2} a_{i}\left(d_{i+1}-\Delta_{i}\right)-2 \beta_{i}{ }^{2} \alpha_{i}\left(\Delta_{i}-d_{i}\right)-2 b_{i}{ }^{2} \alpha_{i} \Delta_{i} \\
& +2 \beta_{i}{ }^{2} \alpha_{i}\left(d_{i+1}-\Delta_{i}\right)+2 b_{i} a_{i} \beta_{i}\left(d_{i+1}-\Delta_{i}\right) \\
& -2 b_{i} \alpha_{i} \beta_{i}\left(\Delta_{i}-d_{i}\right)+2 a_{i} b_{i} \alpha_{i}\left(\Delta_{i}-d_{i}\right) \\
& -2 \alpha_{i}^{2} \beta_{i}\left(d_{i+1}-\Delta_{i}\right)-2 a_{i} \alpha_{i} \beta_{i}\left(d_{i+1}-\Delta_{i}\right) \\
& -2 b_{i}^{2} a_{i} \Delta_{i}+2 \alpha_{i}^{2} b_{i}\left(\Delta_{i}-d_{i}\right)-2 b_{i} \alpha_{i} \beta_{i}\left(\Delta_{i}-d_{i}\right) \\
& +2 a_{i} \alpha_{i} \beta_{i}\left(\Delta_{i}-d_{i}\right)-16 b_{i} \alpha_{i} \beta_{i} \Delta_{i}+16 a_{i} \alpha_{i} \beta_{i} \Delta_{i} \\
& -16 \alpha_{i}^{2} \beta_{i}\left(\Delta_{i}-d_{i}\right)+2 b_{i} a_{i} \beta_{i} \Delta_{i}+16 \beta_{i}^{2} \alpha_{i}\left(d_{i+1}\right. \\
& \left.-\Delta_{i}\right)+2 \alpha_{i}^{2} \beta_{i}\left(\Delta_{i}-d_{i}\right)+2 a_{i} b_{i} \alpha_{i} \Delta_{i}+2 a_{i}^{2} b_{i} \Delta_{i} \\
& -2 a_{i}^{2} \beta_{i}\left(d_{i+1}-\Delta_{i}\right)+2 b_{i} \alpha_{i} \beta_{i}\left(d_{i+1}-\Delta_{i}\right) \\
& +2 b_{i} \alpha_{i} \beta_{i}\left(d_{i+1}-\Delta_{i}\right)-2 b_{i}^{2} \alpha_{i}\left(\Delta_{i}-d_{i}\right) \\
& +2 a_{i} \alpha_{i} \beta_{i}\left(\Delta_{i}-d_{i}\right), \\
& V_{4}=\left(V_{5}+V_{7}-V_{6}\right)+2 a_{i}^{2} \beta_{i} \Delta_{i}-2 a_{i} \alpha_{i} \beta_{i}\left(d_{i+1}-\Delta_{i}\right) \\
& +2 \beta_{i}^{2} a_{i}\left(d_{i+1}-\Delta_{i}\right)-2 \beta_{i}^{2} \alpha_{i}\left(\Delta_{i}-d_{i}\right) \\
& +2 a_{i} \alpha_{i} \beta_{i}\left(\Delta_{i}-d_{i}\right)+2 \beta_{i}{ }^{2} \alpha_{i}\left(d_{i+1}-\Delta_{i}\right) \\
& +2 b_{i} a_{i} \beta_{i}\left(d_{i+1}-\Delta_{i}\right)-2 b_{i} \alpha_{i} \beta_{i}\left(\Delta_{i}-d_{i}\right) \\
& +2 a_{i} b_{i} \alpha_{i}\left(\Delta_{i}-d_{i}\right)+2 a_{i}^{2} b_{i} \Delta_{i}-2 b_{i}^{2} \alpha_{i}\left(\Delta_{i}-d_{i}\right) \\
& -2 a_{i} \alpha_{i} \beta_{i}\left(d_{i+1}-\Delta_{i}\right)-2 b_{i}^{2} a_{i} \Delta_{i}+2 \alpha_{i}^{2} b_{i}\left(\Delta_{i}-d_{i}\right) \\
& -2 \alpha_{i}^{2} \beta_{i}\left(d_{i+1}-\Delta_{i}\right)+16 a_{i} \alpha_{i} \beta_{i} \Delta_{i}+2 b_{i} \alpha_{i} \beta_{i}\left(d_{i+1}\right. \\
& \left.-\Delta_{i}\right)-16 \alpha_{i}^{2} \beta_{i}\left(\Delta_{i}-d_{i}\right)+2 b_{i} a_{i} \beta_{i} \Delta_{i} \\
& -2 b_{i} \alpha_{i} \beta_{i}\left(\Delta_{i}-d_{i}\right)+2 a_{i} \alpha_{i} \beta_{i}\left(\Delta_{i}-d_{i}\right) \\
& -2 a_{i}^{2} \beta_{i}\left(d_{i+1}-\Delta_{i}\right)+2 b_{i}^{2} \alpha_{i} \Delta_{i}-16 b_{i} \alpha_{i} \beta_{i} \Delta_{i} \\
& +16 \beta_{i}^{2} \alpha_{i}\left(d_{i+1}-\Delta_{i}\right)+2 \alpha_{i}^{2} \beta_{i}\left(\Delta_{i}-d_{i}\right) \\
& +2 a_{i} b_{i} \alpha_{i} \Delta_{i}+2 b_{i} \alpha_{i} \beta_{i}\left(d_{i+1}-\Delta_{i}\right), \\
& V_{5}=2\left(V_{6}-V_{7}\right)+6 \beta_{i}\left(a_{i}^{2} \Delta_{i}+a_{i} \alpha_{i}\left(\Delta_{i}-d_{i}\right)\right. \\
& -b_{i} a_{i} \Delta_{i}-b_{i} \alpha_{i}\left(\Delta_{i}-d_{i}\right)-b_{i} \alpha_{i} \Delta_{i} \alpha_{i}^{2}\left(\Delta_{i}-d_{i}\right) \\
& \left.+a_{i} \alpha_{i} \Delta_{i}+2 \beta_{i} \alpha_{i}\left(d_{i+1}-\Delta_{i}\right)\right), \\
& V_{6}=V_{7}+6 \beta_{i}^{2}\left(\alpha_{i}\left(d_{i+1}-\Delta_{i}\right)+a_{i}\left(d_{i+1}-\Delta_{i}\right)\right. \\
& \left.-\alpha_{i}\left(\Delta_{i}-d_{i}\right)\right) \text {, } \\
& V_{7}=2 \beta_{i}^{2}\left(b_{i} d_{i+1}-\Delta_{i}\left(a_{i}+\alpha_{i}\right)+\alpha_{i} d_{i}\right) \text {. }
\end{aligned}
$$

TABle 1: Positive data from Sarfraz et al. [4].

\begin{tabular}{cccccccc}
\hline$i$ & 1 & 2 & 3 & 4 & 5 & 6 & 7 \\
\hline$x_{i}$ & 1 & 2 & 4 & 5 & 7 & 8 & 9 \\
$f_{i}$ & 24.6162 & 2.4616 & 41.0270 & 4.1027 & 57.4378 & 5.7438 & 6 \\
\hline
\end{tabular}

TABle 2: Numerical results for Figure 1(b).

\begin{tabular}{cccccccc}
\hline$i$ & 1 & 2 & 3 & 4 & 5 & 6 & 7 \\
\hline$d_{i}$ & -35.97 & -8.34 & -18.19 & -15.72 & -25.57 & -25.72 & 26.23 \\
$a_{i}$ & 0.74 & 3.4 & 0.23 & 3.84 & 0.23 & 2.25 & - \\
$b_{i}$ & 0.05 & 0.05 & 0.05 & 0.05 & 0.05 & 2.24 & - \\
\hline
\end{tabular}

From (5), $\left(\Delta_{i}-d_{i}\right)>0$, and $\left(d_{i+1}-\Delta_{i}\right)>0$ the following conditions are sufficient for the rational cubic function (2) to preserve convexity:

$$
\begin{aligned}
& a_{i}>\max \left\{0, \frac{\alpha_{i}\left(d_{i}-\Delta_{i}\right)}{\left(d_{i+1}-\Delta_{i}\right)}, \frac{\alpha_{i}\left(d_{i+1}-\Delta_{i}\right)}{\Delta_{i}}\right\}, \\
& b_{i}>\max \left\{0, \frac{\beta_{i}\left(d_{i+1}-\Delta_{i}\right)}{\left(d_{i}-\Delta_{i}\right)}, \frac{\Delta_{i}\left(a_{i}+\alpha_{i}\right)}{d_{i+1}},\right. \\
& \left.\frac{\left(\beta_{i} d_{i+1}+a_{i} d_{i}\right)}{\Delta_{i}}\right\} .
\end{aligned}
$$

The following theorem summarizes the previous discussion.

Theorem 3. Let $\left\{\left(x_{i}, f_{i}\right), i=1,2, \ldots, n\right\}$ be convex data. The rational cubic function given in (2) preserves convexity if the following sufficient conditions are satisfied:

$$
\begin{aligned}
& \alpha_{i}>0, \\
& \beta_{i}>0, \\
& a_{i}=\gamma_{i}+\left\{0, \frac{\alpha_{i}\left(d_{i}-\Delta_{i}\right)}{\left(d_{i+1}-\Delta_{i}\right)}, \frac{\alpha_{i}\left(d_{i+1}-\Delta_{i}\right)}{\Delta_{i}}\right\}, \quad \gamma_{i}>0, \\
& b_{i} \\
& =\epsilon_{i} \\
& \quad+\left\{0, \frac{\beta_{i}\left(d_{i+1}-\Delta_{i}\right)}{\left(d_{i}-\Delta_{i}\right)}, \frac{\Delta_{i}\left(a_{i}+\alpha_{i}\right)}{d_{i+1}}, \frac{\left(\beta_{i} d_{i+1}+a_{i} d_{i}\right)}{\Delta_{i}}\right\}, \\
& \epsilon_{i}>0 .
\end{aligned}
$$

\section{Numerical Examples}

In this section, some numerical examples are illustrated to visualize the results in the previous section.

Example 1. The positive data set in Table 1 is taken from Sarfraz et al. [4]. The curve in Figure 1(a) is produced by using a rational cubic Ball function that does not preserve positivity, whereas Figure 1(b) shows a positive curve that is produced by the positive data interpolation scheme developed in the previous section. Figure $1(\mathrm{c})$ is generated by using rational cubic spline scheme proposed in [1]. One can note that 
TABLE 3: Monotone data set taken from [7].

\begin{tabular}{cccccccccccc}
\hline$i$ & 1 & 2 & 3 & 4 & 5 & 6 & 7 & 8 & 9 & 10 & 11 \\
\hline$x_{i}$ & 0 & 2 & 3 & 5 & 6 & 8 & 9 & 11 & 12 & 14 & 15 \\
$f_{i}$ & 10.0 & 10.0 & 10.0 & 10.0 & 10.0 & 10.0 & 10.50 & 15.00 & 50.00 & 60.00 & 85.00 \\
\hline
\end{tabular}

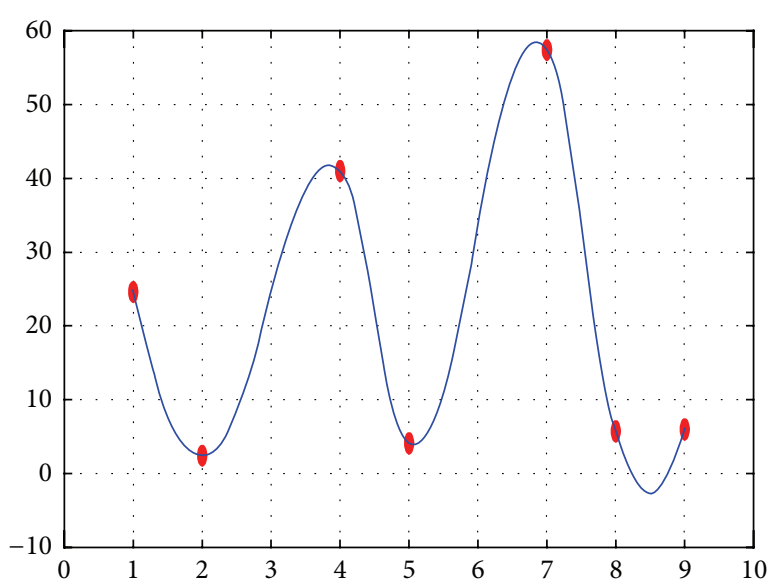

(a)

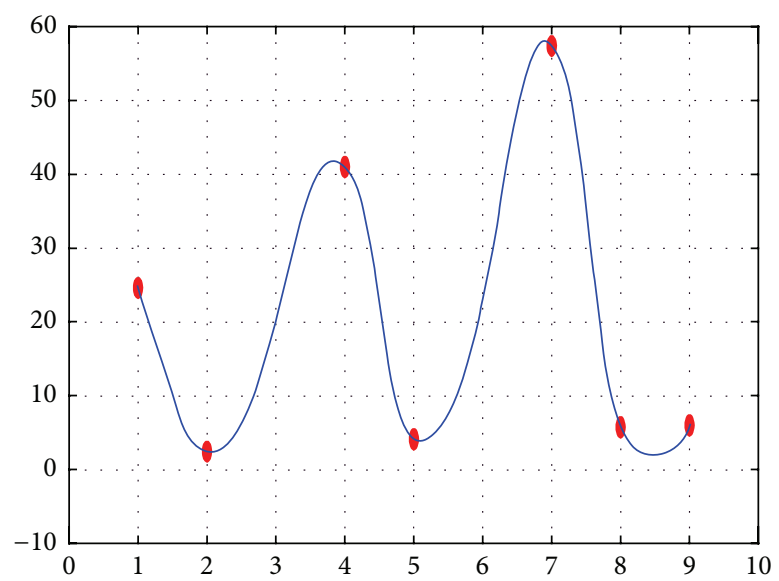

(b)

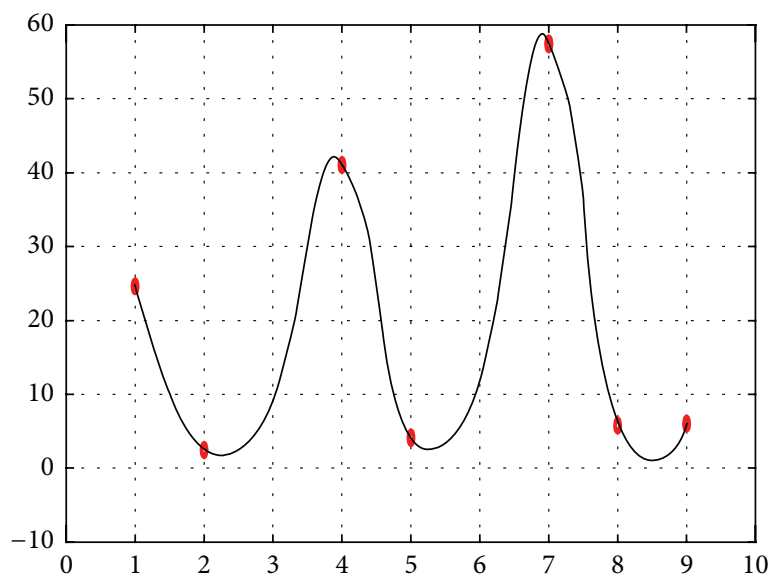

(c)

Figure 1: (a) Rational cubic Ball curve. (b) Positivity preserving interpolation curve with $\alpha_{i}=0.5, \beta_{i}=0.5$. (c) Positive curve using rational cubic spline [1] for data in Table 1.

the curve in Figure 1(b) developed by using positive data interpolation scheme in the previous section is smoother as compared to the curve in Figure 1(c). Table 2 presents some numerical results for Figure 1(b).

Example 2. The monotone data set in Table 3 is presented in Figure 2(a), which shows the plotted curve using a rational cubic Ball function without any constraint. The curve is not monotone, whereas the curve in Figure 2(b), which is produced using the proposed scheme with the same set of data, preserved monotonicity. The monotone curve in Figure 2(c) produced using the rational cubic spline scheme developed in [1]. It is seen that the monotonicity preserving scheme described in the previous section gives a better and smoother curve compared with rational cubic spline scheme
TABle 4: Numerical results for Figure 3(b).

\begin{tabular}{lccccccccccc}
\hline$i$ & 1 & 2 & 3 & 4 & 5 & 6 & 7 & 8 & 9 & 10 & 11 \\
\hline$d_{i}$ & 0.0 & 0.0 & 0.0 & 0.0 & 0.0 & 0.33 & 1.08 & 24 & 25 & 18.3 & 31.7 \\
$a_{i}$ & 0.0 & 0.0 & 0.0 & 0.0 & 0.0 & 0.78 & 0.77 & 0.78 & 1.0 & 0.79 & - \\
$b_{i}$ & 0.0 & 0.0 & 0.0 & 0.0 & 0.0 & 19.2 & 4.3 & 0.37 & 2.5 & 0.48 & - \\
\hline
\end{tabular}

in [1]. Some numerical results for the curve in Figure 2(b) are presented in Table 4.

Example 3. We consider the convex data set in Table 5. The curve in Figure 3(b) is produced by the proposed rational cubic Ball function scheme and shows a convex curve, whereas the curve in Figure 3(a) is produced by the 


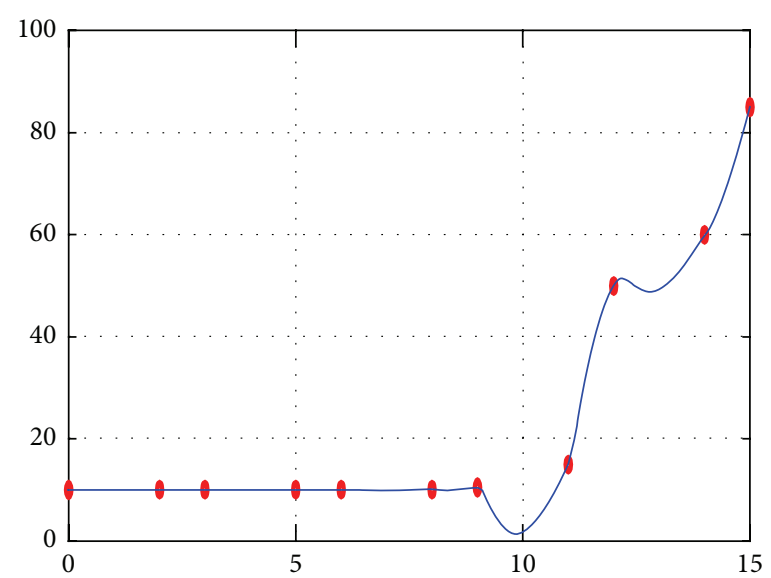

(a)

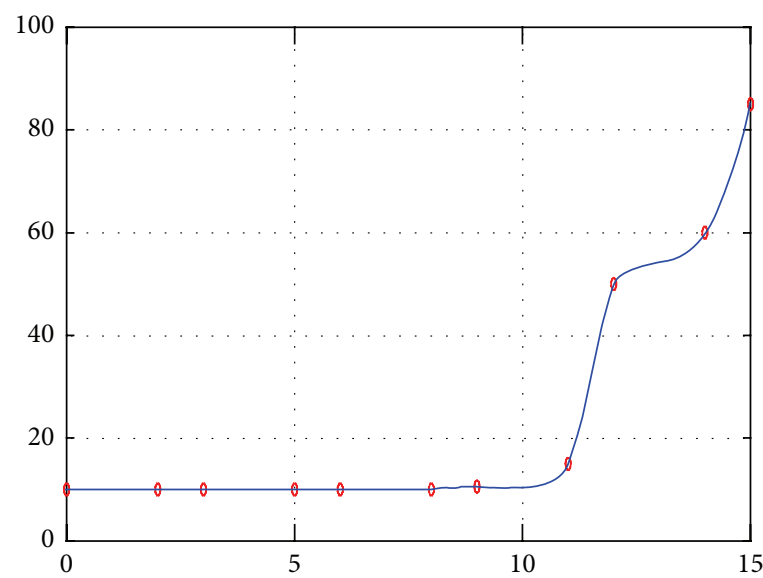

(b)

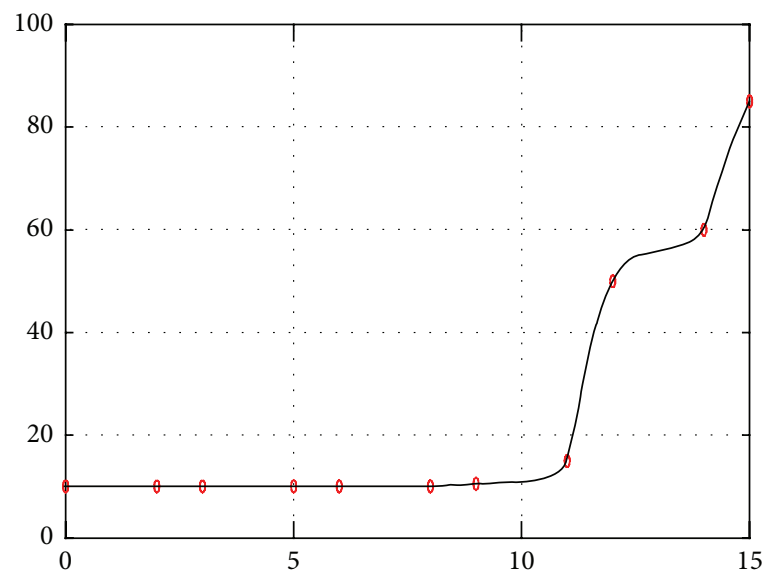

(c)

Figure 2: (a) Rational cubic Ball curve. (b) Monotonicity preserving interpolation curve with $\alpha_{i}=0.05, \beta_{i}=0.5$. (c) Monotone curve using rational cubic spline [1] for data in Table 3.

TABLE 5: Convex data set.

\begin{tabular}{cccccccc}
\hline$i$ & 1 & 2 & 3 & 4 & 5 & 6 & 7 \\
\hline$x_{i}$ & 0 & 2 & 4 & 10 & 28 & 30 & 32 \\
$f_{i}$ & 20.8 & 8.8 & 4.2 & 0.5 & 3.9 & 6.2 & 9.6 \\
\hline
\end{tabular}

rational cubic Ball function described in (2), which does not preserve the shape of the convex data. Figure $3(\mathrm{c})$ is produced by the rational cubic spline scheme developed in [1]. It can be observed that the curve in Figure 3(b) produced by convexity preserving scheme described in the previous section is better and smoother than the curve in Figure 3(c). The shape parameter and the derivative values for the curve in Figure 3(b) are listed in Table 6.

\section{Conclusion}

In this study, a rational cubic Ball interpolant with four free parameters is used to construct a $C^{1}$ interpolant that preserves the shape of positive, monotone, and convex data. Data dependent shape constraints are derived on two of the
TABLE 6: Numerical results for Figure 3(b).

\begin{tabular}{cccccccc}
\hline$i$ & 1 & 2 & 3 & 4 & 5 & 6 & 7 \\
\hline$d_{i}$ & -7.85 & -4.15 & -1.88 & -0.42 & 1.05 & 1.43 & 1.98 \\
$a_{i}$ & 8.50 & 13.59 & 16.40 & 13.86 & 7.52 & 8.50 & - \\
$b_{i}$ & 7.34 & 15.30 & 31.99 & 4.50 & 4.63 & 5.58 & - \\
\hline
\end{tabular}

shape parameters to ensure the preservation of the positivity, monotonicity, and convexity of the data, whereas the other two can assume any positive value, which provides an extra degree of freedom for designers to refine the curve. The three-point difference formula (arithmetic mean) has been used to compute the values of the derivatives. The scheme has been implemented successfully on a number of data sets and a comparison against one rational cubic spline scheme was carried out. The results show that the developed scheme works well for the tested data sets and obtains better and smoother curve. The proposed scheme can be generalized to the surface case, which is under process investigation by the authors. 


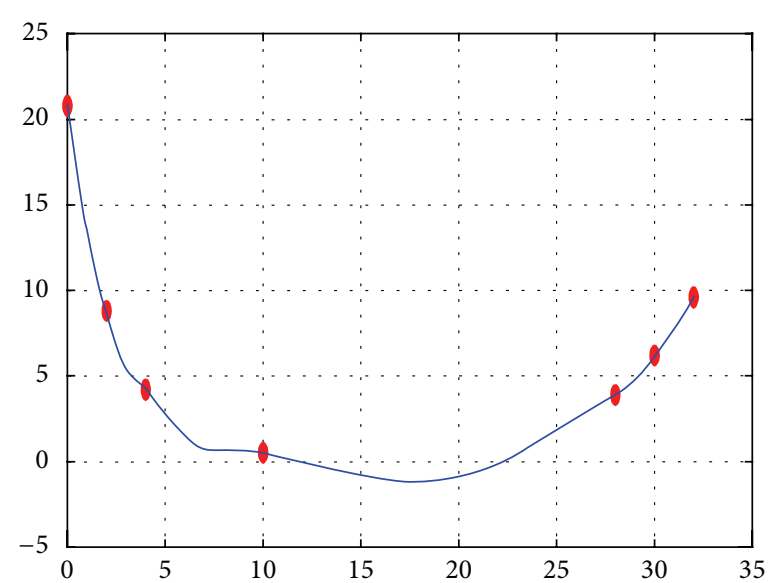

(a)

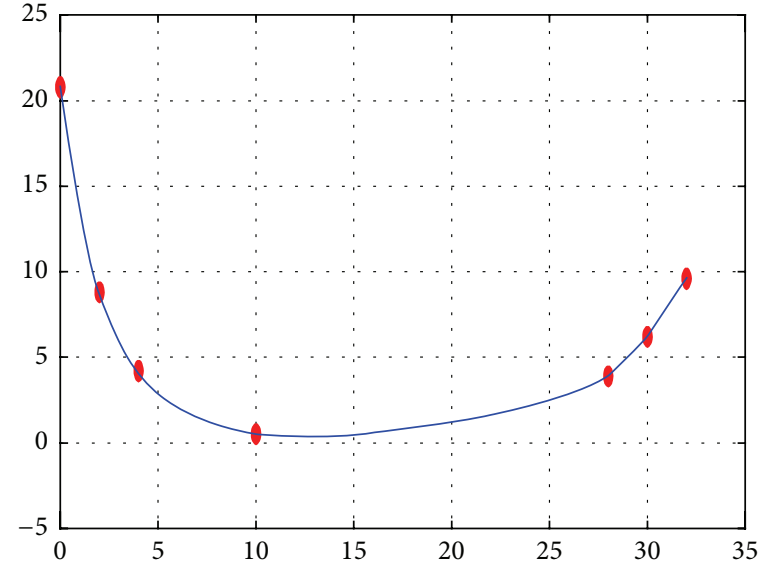

(b)

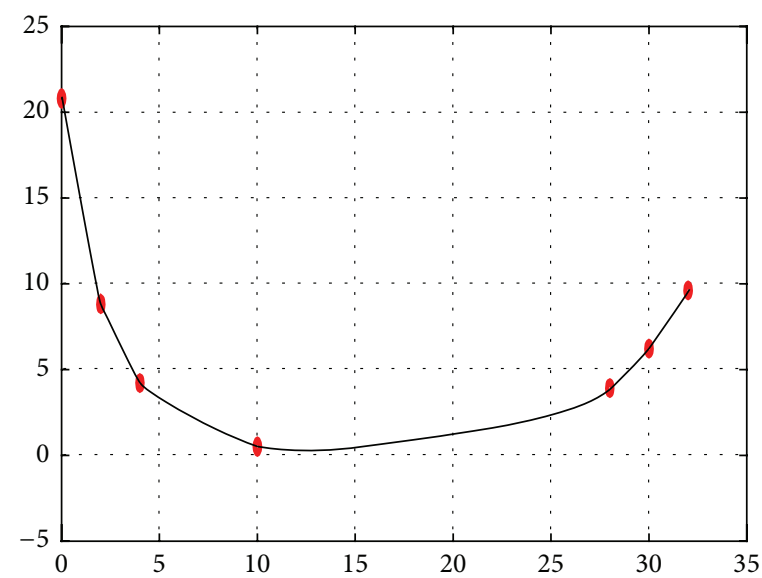

(c)

FiguRE 3: (a) Rational cubic Ball curve. (b) Convexity preserving interpolation curve with $\alpha_{i}=1.5, \beta_{i}=0.5$. (c) Convex curve using rational cubic spline [1] for data in Table 5.

\section{Conflict of Interests}

The authors declare that there is no conflict of interests regarding the publication of this paper.

\section{Acknowledgments}

The authors would like to extend their gratitude to the Ministry of Education of Malaysia and Universiti Sains Malaysia for supporting this work under its Fundamental Research Grant Scheme (FRGS), Account no. 203/PMATHS/6711365.

\section{References}

[1] M. Sarfraz and M. Z. Hussain, "Data visualization using rational spline interpolation," Journal of Computational and Applied Mathematics, vol. 189, no. 1-2, pp. 513-525, 2006.

[2] M. Sarfraz, S. Butt, and M. Z. Hussain, "Visualization of shaped data by a rational cubic spline interpolation," Computers \& Graphics, vol. 25, no. 5, pp. 833-845, 2001.

[3] M. Z. Hussain, N. Ayub, and M. Irshad, "Visualization of 2D data by rational quadratic functions," Journal of Information and Computing Science, vol. 2, no. 1, pp. 17-26, 2007.
[4] M. Sarfraz, M. Z. Hussain, and M. Hussain, "Shape-preserving curve interpolation," International Journal of Computer Mathematics, vol. 89, no. 1, pp. 35-53, 2012.

[5] A. N. H. Tahat, A. R. M. Piah, and Z. R. Yahya, "Positivity preserving rational cubic Ball constrained interpolation," in Proceedings of the 21st National Symposium on Mathematical Sciences (SKSM21 '13), pp. 331-336, AIP Publishing, Penang, Malaysia, November 2013.

[6] M. Z. Hussain and M. Hussain, "Visualization of data preserving monotonicity," Applied Mathematics and Computation, vol. 190, no. 2, pp. 1353-1364, 2007.

[7] M. Z. Hussain and M. Sarfraz, "Monotone piecewise rational cubic interpolation," International Journal of Computer Mathematics, vol. 86, no. 3, pp. 423-430, 2009.

[8] R. Delbourgo and J. A. Gregory, "Shape preserving piecewise rational interpolation," SIAM Journal on Scientific and Statistical Computing, vol. 6, no. 4, pp. 967-976, 1985.

[9] M. Tian, "Monotonicity-preserving piecewise rational cubic interpolation," International Journal of Mathematical Analysis, vol. 5, no. 1-4, pp. 99-104, 2011.

[10] A. R. M. Piah and K. Unsworth, "Improved sufficient conditions for monotonic piecewise rational quartic interpolation," Sains Malaysiana, vol. 40, no. 10, pp. 1173-1178, 2011. 
[11] Q. Wang and J. Tan, "Rational quartic spline involving shape parameters," Journal of Information and Computational Science, vol. 1, no. 1, pp. 127-130, 2004.

[12] A. R. M. Piah and K. Unsworth, "Monotonicity preserving rational cubic ball interpolation," In press.

[13] T. S. Shaikh, M. Sarfraz, and M. Z. Hussain, "Shape preserving positive and convex data visualization using rational bi-cubic functions," Pakistan Journal of Statistics \& Operation Research, vol. 8, no. 1, pp. 121-138, 2012.

[14] K. W. Brodlie and S. Butt, "Preserving convexity using piecewise cubic interpolation," Computers \& Graphics, vol. 15, no. 1, pp. 1523, 1991.

[15] M. Sarfraz, "Convexity preserving piecewise rational interpolation for planar curves," Bulletin of the Korean Mathematical Society, vol. 29, no. 2, pp. 193-200, 1992.

[16] M. Sarfraz, "Visualization of positive and convex data by a rational cubic spline interpolation," Information Sciences, vol. 146, no. 1-4, pp. 239-254, 2002.

[17] M. Hussain, M. Z. Hussain, and M. Sarfraz, "Data visualization using spline functions," Pakistan Journal of Statistics \& Operation Research, vol. 9, no. 2, pp. 181-203, 2013.

[18] A. A. Ball, "CONSURF. Part one: introduction of the conic lofting tile," Computer-Aided Design, vol. 6, no. 4, pp. 243-249, 1974.

[19] W. Guojin, "Ball curve of high degree and its geometric properties," Applied Mathematics, vol. 2, no. 1, pp. 126-140, 1987.

[20] H. B. Said, "A generalized Ball curve and its recursive algorithm," ACM Transactions on Graphics, vol. 8, no. 4, pp. 360-371, 1989.

[21] T. N. T. Goodman and H. B. Said, "Properties of generalized Ball curves and surfaces," Computer-Aided Design, vol. 23, no. 8, pp. 554-560, 1991.

[22] T. N. T. Goodman and H. B. Said, "Shape preserving properties of the generalised Ball basis," Computer Aided Geometric Design, vol. 8, no. 2, pp. 115-121, 1991. 


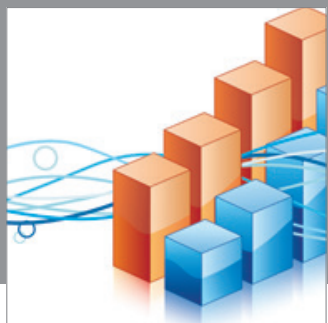

Advances in

Operations Research

mansans

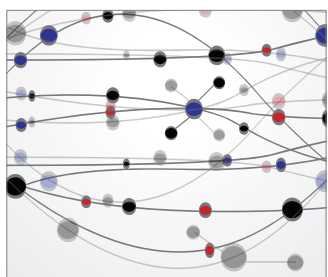

The Scientific World Journal
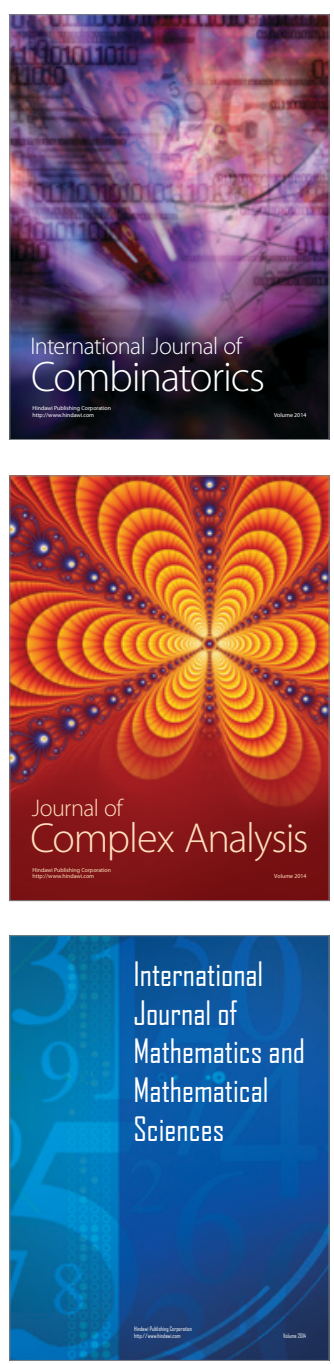
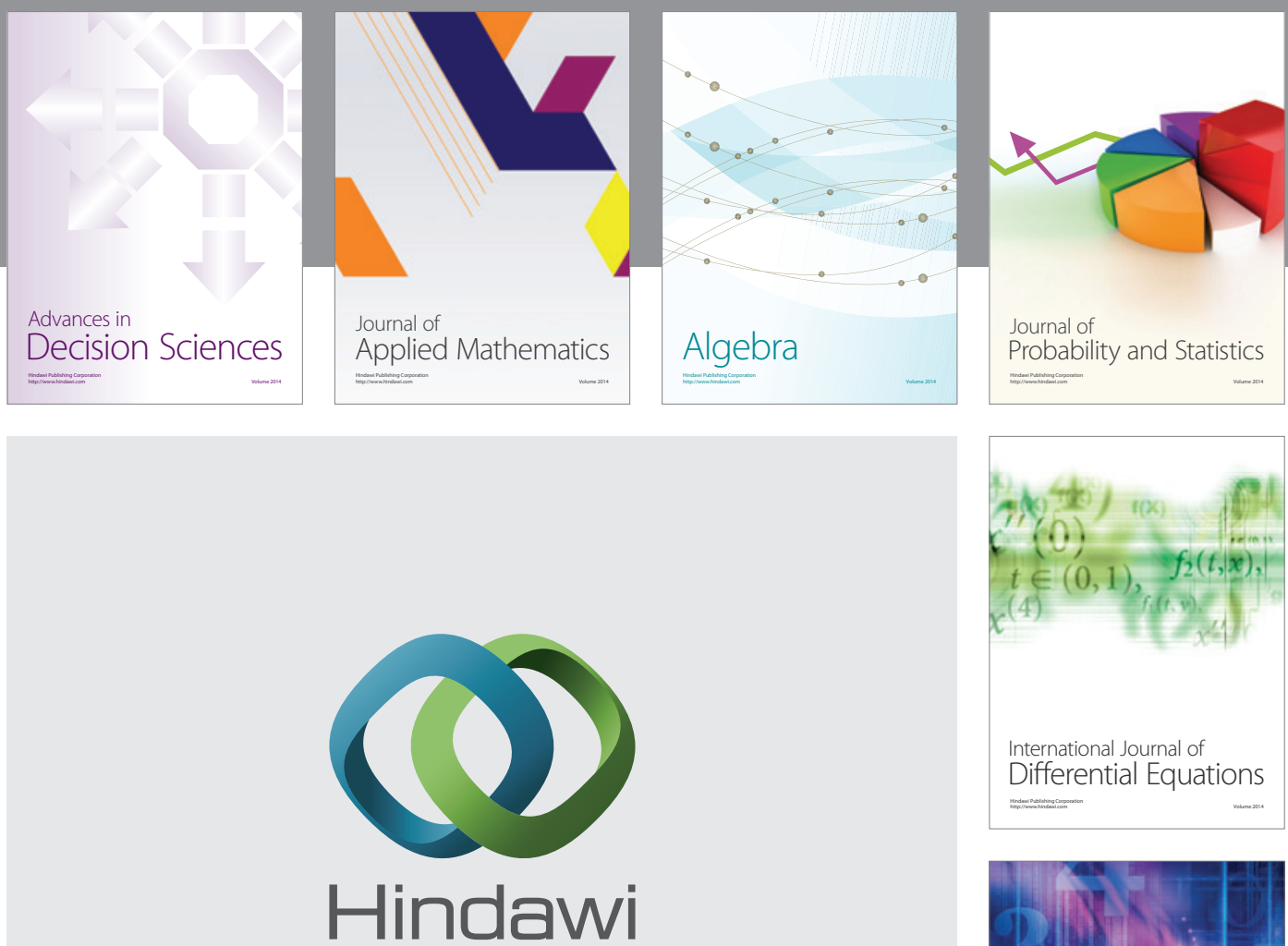

Submit your manuscripts at http://www.hindawi.com
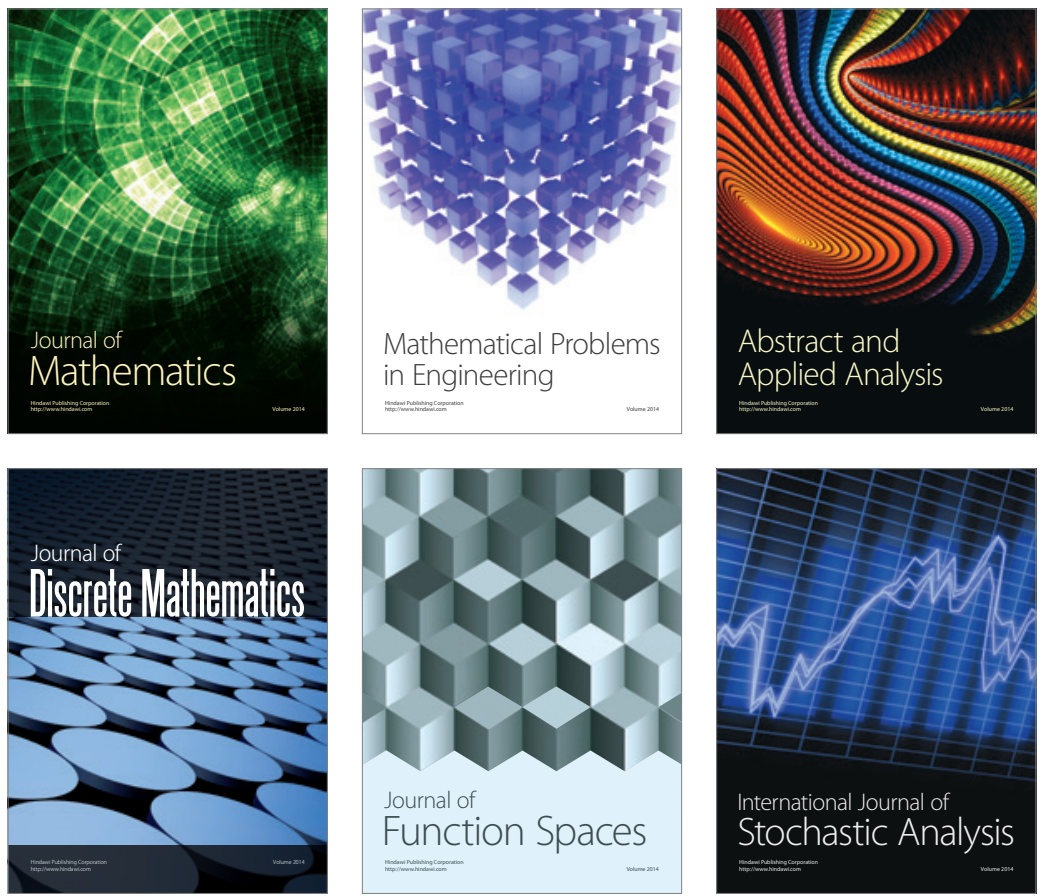

Journal of

Function Spaces

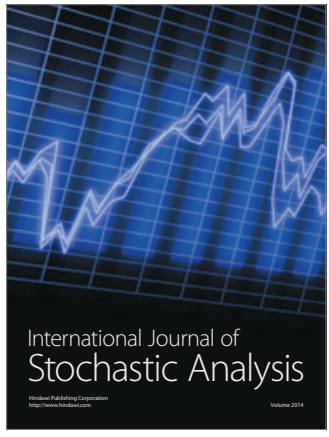

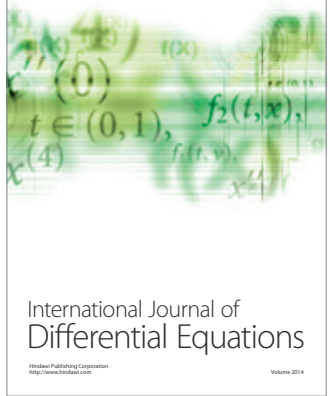
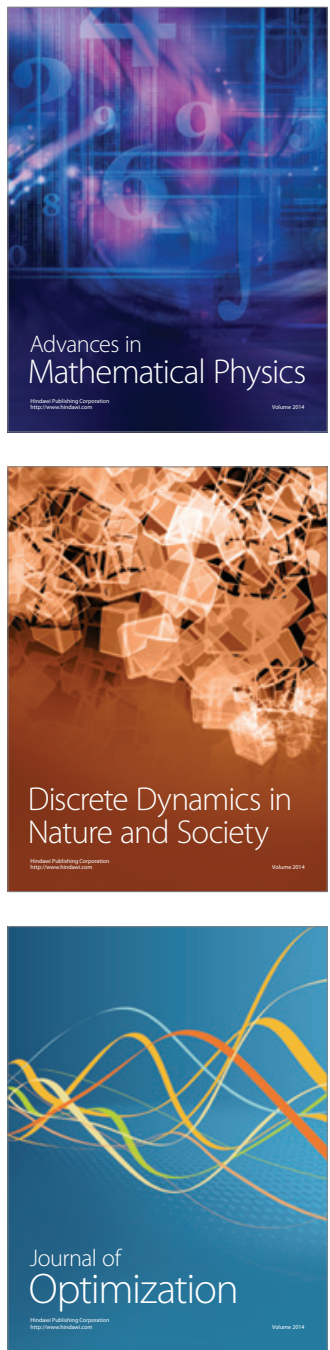